\section{MCRPC: positives CHMP-Votum für Enzalutamid}

— Der Ausschuss für Humanarzneimittel (CHMP) der Europäischen Arzneimittelagentur (EMA) hat Ende April die Zulassung von Enzalutamid zur Behandlung von erwachsenen Männern mit metastasiertem kastrationsresistentem Prostatakarzinom, deren Erkrankung während oder nach DocetaxelTherapie fortschreitet, empfohlen.

Enzalutamid ist ein neuer Androgenrezeptor-Signalinhibitor, der einmal täglich oral eingenommen wird. Er hemmt mehrere Schritte des Androgenrezeptor-Signalwegs, reduziert so das Wachstum der Karzinom- zellen und induziert das Absterben maligner Zellen. Das positive CHMP-Votum basiert auf den Ergebnissen der Phase-III-Zulassungsstudie AFFIRM, die belegt, dass Enzalutamid im Vergleich zu Placebo zu einer statistisch signifikanten Verlängerung ( $p<0,0001$ ) des Gesamtüberlebens führt, mit einem medianen Überleben von 18,4 Monaten in der Enzalutamid-Gruppe im Vergleich zu 13,6 Monaten in der PlaceboGruppe - ein Überlebensvorteil von 4,8 Monaten [Hazard Ratio (HR) $=0,63$ ]. Die Studie zeigte außerdem, dass Enzalutamid von den Patienten allgemein gut vertragen wurde und alle sekundären Endpunkte erreicht hat [Scher HI et al. N Engl J Med 2012; 367: 1187-97].

Die Empfehlung des CHMP wird von der Europäischen Kommission, die die Befugnis zur Zulassung von Arzneimitteln in der europäischen Union hat, überprüft. Der Bescheid der Europäischen Kommission ergeht üblicherweise innerhalb von 60 Tagen nach einer positiven CHMP-Empfehlung.

Nach Informationen von

Astellas, München

\title{
Idiopathische OAB: Botulinumtoxin lähmt die Blasenmuskulatur
}

_ Für erwachsene Patienten mit idiopathisch überaktiver Blase (OAB) mit den Symptomen Harninkontinenz, imperativem Harndrang und Pollakisurie, die nicht ausreichend auf Anticholinergika ansprechen beziehungsweise diese nicht vertragen, gibt es mit Botulinumtoxin A (Botox ${ }^{\circledast}$ ) eine neue Therapieoption. 100 Einheiten $(10 \mathrm{ml})$ des Medikaments werden in Fraktionen von je $0,5 \mathrm{ml}$ mithilfe eines Zystoskops an 20 Stellen - unter Aussparung des Trigonums - in den Detrusormuskel injiziert (Abstand $1 \mathrm{~cm}$, Tiefe etwa $2 \mathrm{~mm}$ ). Im Vergleich zu Placebo reduziert diese Therapie signifikant Harninkontinenzepisoden, Harndrang, Miktionsfrequenz und steigert die Lebensqualität betroffener Patienten.

Die Anfang 2013 erfolgte Zulassung basiert auf internationalen Phase III-Studien mit über 1.100 anticholinergisch vorbehandelten Patienten, die durchschnittlich mehr als fünf Harninkontinenzepisoden hatten. "Unter der Behandlung zeigte sich bereits in Woche 2 eine hochsignifikante Reduktion der täglichen Inkontinenzepisoden um 51\%, die im Verlauf anhaltend war", berichtete Prof. Klaus-Peter Jünemann, Kiel. 27,1\% der Patienten waren nach zwölf Wochen komplett kontinent (Placebo 8,4\%), bei $60 \%$ halbierte sich die Zahl der Episoden. Harndrang, Miktionsfrequenz und Nykturie verringerten sich ebenfalls signifikant. Als häufigste Nebenwirkungen wurden Harnwegsinfekte und Dysurie beschrieben. Die Abbruchrate lag unter $2 \%$.
Daten zur Lebensqualität zeigen klinisch relevante Verbesserungen in allen Domänen des I-QOL (Incontinence Quality Of Life Questionnaire) um über 20 Punkte sowie im KHQ (King's Health Questionnaire) um bis zu 25 Punkte. Über $60 \%$ der Patienten beurteilten ihre Symptome bereits nach zwei Wochen und über die Studiendauer von zwölf Wochen als "deutlich verbessert" oder „verbessert".
Botulinumtoxin hemmt reversibel die Freisetzung von Acetylcholin und anderen Neurotransmittern aus cholinergen Nervenenden und verringert so die Aktivität des Blasenmuskels. Die Wirkung hält bis zu zwölf Monate an, die Behandlung mit Botulinumtoxin A kann nach mindestens zwölf Wochen wiederholt werden. Die Behandlung mit Botulinumtoxin $A$ ist bei $O A B$ voll erstattungsfähig. Michael Koczorek

Pressegespräch „Zulassung von BOTOX ${ }^{\circledR}$ bei überaktiver Blase", Hamburg, 17. April 2013 Veranstalter: Allergan, Ettlingen

\section{PSA-Schnelltest ermöglicht Messungen direkt in der Praxis}

- Ein neuer quantitativer PSA-Schnelltest für die patientennahe Diagnostik erleichtert jetzt das PSA-Screening für den niedergelassenen Urologen. Der neue Test wurde in einer klinischen Studie an der Universität Tübingen evaluiert. Dazu wurden bei 377 Patienten die PSA-Werte mit einem Immulite 2000 Laborsystem gemessen und zusätzlich mit dem quantitativen Schnelltest CancerCheck ${ }^{\circledast}$ PSA bestimmt. Der Schnelltest liegt als LateralFlow-Assay in einer Testkassette vor und wird mittels eines kleinen POC-Readers (concile ${ }^{\circledast} 100$ ) ausgewertet. Das POCSystem aus Reader und quantitativem PSA-Test zeigte eine sehr gute Überein- stimmung mit dem Laborsystem ohne signifikante Abweichungen. Der PSASchnelltest identifizierte 83 von 89 tumorfreien Patienten richtig negativ und 233 von 288 Patienten mit Prostatakarzinom richtig positiv. Spezifität und Sensitivität betrugen somit $93 \%$ und $81 \%$.

Der Schnelltest bietet schnelle und verlässliche PSA-Messungen in der urologischen Praxis. Das Ergebnis liegt nach 20 Minuten vor und ist somit zeitnah zum Patientengespräch verfügbar. Der quantitative PSA-Schnelltest ist als In-vitro-Diagnostikum CE-zertifiziert.

Nach Informationen von Concile, Freiburg 